\title{
Classification and Selection of Prototyping Activities for Interaction Design
}

\author{
Stefano Filippi, Daniela Barattin \\ Product Innovation Research Group, Electrical, Management and Mechanical Engineering Department, \\ University of Udine, Udine, Italy \\ Email: filippi@uniud.it
}

Received April 19, 2012; revised May 28, 2012; accepted June 29, 2012

\begin{abstract}
Because of the evolution of markets and technologies, prototyping concerns should be kept updated almost day by day. Moreover, user centered design moves the focus towards interaction issues. Prototyping activities matching such characteristics are already available, but they are not so diffused in the industrial domain. This is due to many reasons; an important one is that a rigorous classification of them is missing, as well as an effective helping tool for the selection of the best activities, given the design context. The research described in this paper aims at defining a new classification of prototyping activities, as well as at developing a selection algorithm to choose the best ones in an automatic way. These goals are pursued by defining a set of characteristics that allow describing accurately the prototyping activities. The resulting classification is made by five classes, based on eighteen characteristics. This classification is exploited by the first release of an algorithm for the selection of the best activities, chosen in order to satisfy design situations described thanks to a different set of eleven indices. Five experiences in the field have been used up to now as a starting point for validating the research outcomes.
\end{abstract}

Keywords: Prototyping Activities; Interaction Design; Virtual Reality; Augmented Reality

\section{Introduction}

Current mechatronic devices show large amount of hitech components, as well as an increasing number of functionalities. For this reason, interaction issues become more and more important, and users should be the center of the development process. Moreover, competition is increasing day by day and this implies that costs and time must be minimized. All of this drives to the need of revising the classic development processes, by considering more than ever the central role of interaction design tools and methods. Anyway, this is not enough; together with this attention to usability issues, some care must be taken about prototyping activities. Usability evaluation should be performed more than once during the development process, in order to validate the product interaction with users and environment.

Prototyping methods and tools focused on usability issues have evolved as well, mainly thanks to the virtual reality concept. It allows modeling and testing product functionalities at much lower costs than before; anyway, the absence of realness remains with no doubt a big drawback. In order to partially solve this problem, mixed approaches have been developed. Real and virtual components are used together during prototyping activities. The exploitation of all of this in the industrial field is quite limited at the moment, because of costs and im- plementation difficulties. Moreover, industries and research laboratories are somehow confused by the huge amount of methods and tools on the market, and the absence of a real classification to be exploited in selecting the most suitable prototyping activities does not help.

For these reasons, the research described in this paper has two main goals. First, it aims at generating a new classification of prototyping activities by defining real, measurable dimensions. Then, the development of a selection algorithm takes place. This algorithm allows selecting the best activities automatically, given the description of the scenario where the usability evaluation will take place.

The paper runs as follows. After some background about interaction design, virtual/augmented reality, and existing prototyping activities, the definition of the dimensions used to classify these activities is described. Then, all the steps up to the generation of the selection algorithm are presented. Next paragraph deals with some experiences in the field, used as a starting point for the validation of the proposed approach to interaction prototyping. Some conclusions and suggestions for future work close the paper.

\section{Background}

This paragraph describes the two main topics involved in 
this research: interaction design and virtual/augmented reality. The first one refers to the application field where the methods and tools of the second one are exploited.

\subsection{Interaction Design}

Interaction design - ID - focuses on the correct interpretation and implementation of the user-product dialogue [1]. It allows generating products ready to be easily and intuitively used by the most of the users, and accepted since the beginning, thus avoiding soft reliability problems [2]. It is quite young as a research field, but already full of methods and tools based on the usability concept. As the ISO 9241 standard says, usability is "the effectiveness, efficiency and satisfaction with which specified users achieve specified goals in particular environments" [3]. Specifically, ID methods and tools focus on user satisfaction because new definitions of product quality are heavily based on it. The first developed tools were collections of principles and guidelines, mainly used for usability evaluation activities. Some examples of them are the seven dialogic ISO principles [4], the eight Shneiderman's golden rules [4], the ten Nielsen's heuristics [5] and the interaction paradigms of Dix, Finlay, Abowd and Beale [6].

The most interesting methods couple the analysis of the users' needs with tools to generate design solutions starting from them. Some examples are the interdisciplinary approach with a user-system focus [2], the user action framework [7] and the intent method [8].

\subsection{Virtual/Augmented Reality}

Virtual reality - VR - is a powerful tool to simulate the presence of a product and the interaction with it in a virtual environment [9]. Many applications appeared on the market implementing these characteristics. These applications satisfy the requirements coming from different domains, from videogames to mobile communications [10], to industrial and engineering design [11], up to furnishing configuration [12], etc. Directness and interactivity are the most interesting characteristics of VR. Elements inside the virtual environment can be easily customized by changing locations, orientations, colors, etc.; moreover, in particular situations the real interaction between users and product models is allowed.

The technological innovations enhance the realness of the interaction with virtual environments. For example, head-mounted displays give the users a real 3D view of the product models and environments; haptic devices as data gloves allow advanced feedbacks [13].

Recently, some industries, especially those ones related to furnishings, clothing, etc., have been looking for something that would avoid the inner problem of VR, the absence of realness (unless exploiting very expensive technologies). So, augmented reality-AR — has come to the stage. AR, defined by Azuma as "the real-time combination of virtual and real elements", allows integrating virtual components, images, 3D models, etc., in a real scene, generating in this way new interaction capabilities [14]. This approach presents many advantages if compared to VR: it manages a continuous visual reference to the real users' context, avoiding any sensation of getting lost; it is very intuitive and user friendly to adopt, because user's skill and/or knowledge of CAD applications are not required; by using it, users are allowed catching their goals in a faster and more satisfactory way; the product models integrated in the real world can maintain full interactivity with the users; finally, AR applications are often very cheap, because pieces of software, libraries, etc. can be found over the web free of charge.

Other approaches than VR and AR are used in prototyping activities focused on usability evaluation. Especially when dealing with small products, because of technological limitations, images are projected on physical supports. The shape of these supports is similar or equal to the final product one and thanks to them the interaction gains in realness. All of this is named mixed reality [15]. The augmented virtuality is another approach coming from VR. It consists in virtually representing the environment where a real, physical prototype is placed and tested [16]. It is not so diffused at the moment because of technological costs. The augmented virtuality is usually adopted when hazardous environments as radioactive fields are involved, or when it is very difficult to find or reach the environments for any reason.

\subsection{Prototyping Activities}

Prototyping activities considered in this research comprehend both technologies and procedures to build and exploit prototypes in the product development process. The first example of them can be found in the computer numerical control $-\mathrm{CNC}-$ machines. They are the typical equipment extensively used in industrial domains [17]. In order to make prototyping activities more affordable, rapid prototyping technologies appeared on the market in the last two decades. The building approach is exactly opposite to the CNC machine one. In rapid prototyping, parts are generated by adding material instead of removing it. Some examples of RP technologies are stereolitography, direct metal laser sintering, laminated object manufacturing and 3D printing [18]. The activities described up to now always generate physical prototypes. The exploitation of virtual reality, increased together with the power of computers, caused that physical prototypes started to be substituted with digital mock-ups, product representations much cheaper than the other ones, easier to generate, manage, store and retrieve, share, etc. 
[19]. Digital mock-ups allow evaluating the products they represent from many points of view, by much more people than before, and in a wider range of application domains.

\section{Activities}

The research described in this paper is divided in two main stages. The first one is focused on a new classification of existing prototyping activities; the second one aims at developing a selection algorithm given specific design contexts.

\subsection{Prototyping Activity Classification}

The prototyping activity classification comes through a tree-step procedure. Existing classifications described in literature are analyzed first; then, starting from them, a set of characteristics is defined. Finally, this set is exploited for the generation of the new classification.

\subsubsection{Analysis of Existing Classifications}

The classification presented hereafter is based on a set of dimensions that allow describing prototyping activities in detail. These dimensions derive from the analysis of existing classifications in literature. Four approaches have been considered. In [14], Azuma defines the AR concept and its possible exploitation. The main topic of this definition consists in virtualizing part of the environment where the user interacts with the product. So, in this case, two dimensions are important: user and environment. The environment is virtualized from the visual point of view; the other senses as touch and hearing are ignored. In [16], Milgram and Kishino base their classification on the difference between virtual and real, from several points of view. In particular, environment is the main dimension used to classify existing prototyping activities. In this case, the environment concept comprehends also the user and the prototype and this could generate problems because too different entities are managed as a single one. In [20], Bruno et al. propose a description of mixed reality to be exploited in prototyping activities. They consider the environment as fully virtual, while the product is real. They exploit video tracking to implement their prototyping scenarios, but the dimensions used to classify different types of prototyping activities are limited to environment and prototype; user and interaction are ignored. Finally, Bordegoni et al. [21] perform a deep analysis on the exploitation of mixed reality in prototyping, by introducing two new dimensions: kind of the prototype and interaction. Thanks to these dimensions, they derive a new classification framework based also on user type. In this case, environment is not involved in the classification.

\subsubsection{Definition of Dimensions}

The analysis of existing classifications highlights that one of the major problems is the absence of clearly defined and commonly recognized/accepted dimensions to base the classification on. Then, starting from all the acquired knowledge from the literature, a new set of dimensions is developed here. The criteria used for their definition are as follows.

1) Orthogonality. Dimensions must be complimentary each other, without duplications or partial overlapping;

2) Coverage. Dimensions must be related to classic prototyping activities, but they must deal with the several ID and usability evaluation issues as well;

3) Clarity. Dimensions must be stated without misunderstandings, thanks to discrete, assignable values.

By applying these three criteria, the result consists in the following four dimensions, each of them with its specific discrete values.

- Product representation-prototype. It is very important to have the prototype kind as a dimension. It can have two values: real and abstract. The real prototype is the physical one, which can be more or less completed in its parts. The abstract prototype is a product model generated and managed thanks to software applications.

- Environment. This dimension represents all the items needed to perform the interaction, except for users and products. Even in this case, two values are allowed: real and simulated, with the same meaning as the previous case.

- User. The user is the main entity interacting with the prototype. User can be real or simulated. In the first case he/she is directly involved in prototyping activities; in the second case software applications simulate his/her presence and behavior.

- Interaction. Interaction represents the way the user is put into relationship with the product. Interaction can be direct or indirect. Unlike the classic definition of direct or indirect interaction [22], in this case interaction is defined as direct if it is exactly the same as the one expected with the final product. Indirect interaction happens when it requires actions and/or generates feedback different from the expected/designed ones.

- Time. Time represents the phase of the design process when prototyping activities happen. Different phases of the design process require evaluating different aspects of the product; as a consequence, the prototyping activities may differ. In this case, the assignable values correspond to the stages of the product development process.

\subsubsection{Deriving the New Classification}

Prototyping activities are classified into classes thanks to the dimensions described in the previous paragraph. Be- 
fore this, an ordering criterion must be defined. It is based on the importance that dimensions have regarding prototyping. The most important dimension is the environment, given that all the prototyping activities happen inside it. Moreover, three out of the four analyzed studies already consider environment as a dimension and one of them uses it as the sole dimension, even if the concept is intended in a wider way. The second dimension is prototype, the object coming directly from the design process. These two dimensions allow identifying four classes, as shown in Figure 1. User and interaction do not contribute at this main level, but they are important because they allow going deeper in classifying prototyping activities inside the four classes. Finally, there are some cases where environment and/or prototype and/or user are real and simulated/abstract at the same time, for example when an image is superimposed to a physical mock-up. For these situations, a fifth class is provided.

Regarding the fifth dimension, in order to avoid a time-dependent classification that would lead to a too complex management, a static classification has been preferred at the moment. This classification is localized at the end of the concept generation phase, before that concept evaluation activities take place. This is a very important milestone, because future manufacturing costs heavily depend from it.

The description of the five classes is as follows. For each of them, some examples of implementations are highlighted; moreover, the references used in the previous paragraphs and dealing with specific methods/systems are classified as well.

- Real prototyping - RP. Environment is real as well as prototype. No virtualization is present. This is the classic situation. Many technologies have been already developed in order to satisfy the physical prototyping requirements, starting from $\mathrm{CNC}$ milling machines up to rapid prototyping approaches, like stereolitography, etc. [18,23,24].
- Augmented real prototyping-ARP. Product models are placed in real scenes; environment is real and prototypes are completely abstract. There are many examples, like furniture set up [25], visual browsers [26], historical reconstructions of ancient monuments [27], and design of infrastructures and roads [28].

- Augmented virtual prototyping-AVP. Real prototypes are placed in a simulated environment. This approach is not so much widespread at the moment; it is used for very special situations. An example could be the flight simulators [29].

- Virtual prototyping-VP. In this case nothing is real. Environment is simulated and prototype is abstract. Some examples are CAD software packages for mechanical design, like AutoCAD [30] and Blender [31, 32], software packages for design [9] and prototyping [11] processes, operative systems for cell phones and notebooks [10].

- Mixed prototyping-MP. This last class has been generated to manage situations where prototype and/ or environment and/or user could be real and simulated/abstract at the same time. An example of prototyping activity belonging to this class can be found in first aid training courses. Environment is real, as well as dummies. But, thanks to head mounted displays, students can see possible accident effects on them [33]. Another example of user testing during prototyping activities can be found in [15].

\subsection{Development of the Selection Algorithm}

After the definition of the new classification, it is the time to work about the algorithm to select the best prototyping activities for specific design contexts. Prototyping activities are described first, by defining some characteristics. Then, prototyping situations are described as well. In the end, all of them are put into relationship in order to make the best selection case by case.

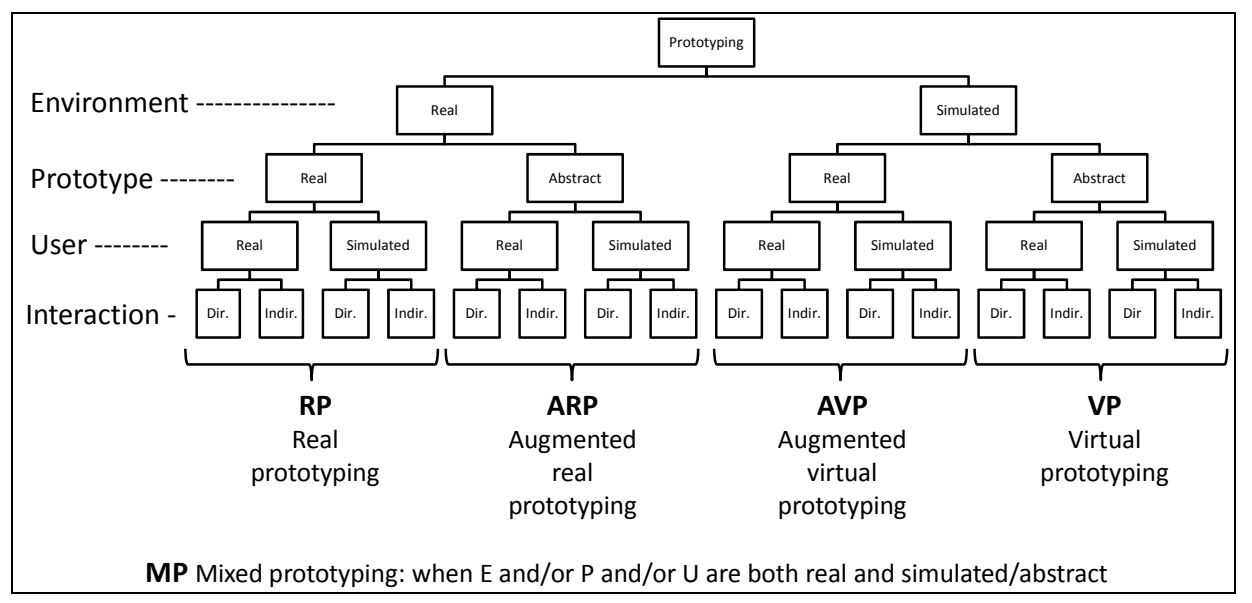

Figure 1. Classification of prototyping activities. 


\subsubsection{Characterizing Prototyping Activities}

Five dimensions defined were enough to generate the classification; however, prototyping activities need to be described more accurately to allow their selection at best. For this reason, the same analysis of literature described in Section 2.1 is exploited here, but with a different goal. The effort is towards the generation of a wider set of references, covering more aspects of prototyping activities. These references are named characteristics. Regarding the literature analysis, unfortunately the virtual component is rarely considered together with the real one. Most of the cases are focused on methods able to classify and select physical prototyping activities [34-37]. On the other hand, sometimes prototyping activities containing virtual/augmented/mixed reality are described with interesting examples, but they are not focused on prototyping [38-42]. For these reasons, the analysis has been quite long and difficult. Anyway, by applying the precise criterion that characteristics must be always measurable, in the end eighteen of them have been highlighted. They are collected in Table $\mathbf{1 .}$

\subsubsection{Enriching the Prototyping Activity Classes with Characteristics}

The characteristics allow describing prototyping activities in a suitable way for successive exploitation by the selection algorithm. Five tests are proposed to three experts in ID and usability evaluation, in order to associate these characteristics to the classes. These tests represent everyday prototyping situations, and the experts are asked to give the characteristics some values, representing how well a specific class could suit for the situations under investigation. The allowed range for values is $[0,1]$; higher the value, more suitable the class. In order to get a set of data as meaningful and objective as possible, a questionnaire made by eight multiple-choice questions is used. This way, collected data can be analyzed consistently and numerical values can be assigned based on averages. Every tests have been evaluated at the end of the concept generation phase, in order to keep valid the limitation described before.

The tests deal with the following prototyping needs.

1) Usability testing of the display and control panel of a washing machine;

2) Testing of user friendliness and affordance of an air purifier used in hazardous environments;

3) Usability testing focused on ergonomic issues, of a PC keyboard;

4) Affordance evaluation of some functions of a new PC operative system;

5) Aesthetical evaluation of watch models on the wrist of possible customers directly by the manufacturer's website.

The results show that experts' judgments agree, even if they performed their work autonomously. First need is solved using MP, because the prototype needs to be real and abstract at the same time. The realness is needed to get direct interaction; the abstraction makes prototype

Table 1. Characteristics of prototyping activities.

\begin{tabular}{|c|c|c|}
\hline Characteristic & Description & Unit \\
\hline Direct interaction & Presence of direct interaction with the prototype & Bool. \\
\hline Physical prototype & Prototype must be real, physical & Bool. \\
\hline Physical feedback & Amount of physical feedback that system gives the user & \# \\
\hline Visual and audio feedback & Amount of feedback that system shows to user as notes, earcon, etc. & \# \\
\hline Real environment & Environment where prototyping activities take place must be real & Bool. \\
\hline $\begin{array}{l}\text { Functions for error recognition and } \\
\text { recovery }\end{array}$ & $\begin{array}{l}\text { Presence of systems that can recognize when error happen and performing error } \\
\text { recovery }\end{array}$ & \# \\
\hline Functions implemented & Functionalities implemented inside the prototype & \# \\
\hline Time required for updates & Time required to create a variation on structure or functionalities of prototype & Hour \\
\hline Cost required for updates & Cost to modify the prototype & $€$ \\
\hline Cost for tooling & Cost for tooling used during prototyping & $€$ \\
\hline Cost for prototyping & Cost to manage and use prototyping facilities & $€$ \\
\hline Cost for set up & Cost for setting up the prototyping process & $€$ \\
\hline Cost for working team & Cost for human-resources & $€$ \\
\hline Human operators & Required people with special skill and knowledge & \# \\
\hline Prototype building time & Time for complete generation of prototype & Hour \\
\hline Environment building time & Time to replicate environment where usage of prototype takes place & Hour \\
\hline Dimensional and geometric accuracy & Required dimensional and geometric tolerances for evaluating prototype correctly & $\mu \mathrm{m}$ \\
\hline
\end{tabular}


modification or substitution easier, in order to gain flexibility in the design process. AVP is selected to satisfy the second need, to perform the evaluation in a simulated, safe environment instead of the hazardous one. The ergonomic issues of the third need are thought to be best evaluated using RP, where stereolitography could generate the physical prototype of the keyboard. The fourth need is managed with VP, because product under evaluation does not show any physical component. ARP is preferred in satisfying the fifth need, because it allows a fast switching of the prototype and in the meantime the real environment, the customer's wrist, is exploited.

The averages of the values expressed by the three experts are collected in Table 2. The rows represent the characteristics and the columns are the classes of prototyping activities.

\subsubsection{Describing Prototyping Situations}

The term prototyping situation is introduced as a way to describe the needs that could happen in a specific moment of the product development process, from the prototyping point of view. They represent the requirements that determine the selection of the best prototyping activities. Prototyping situations are described by mean of indices, generic enough to be exploited in a set of application fields as wide as possible.

Some questionnaires are created to collect the information regarding typical development environments: industrial R\&D departments, research labs, etc. They are focused on collecting the most important needs during design processes, as typical timing for prototyping, prototyping technologies used to evaluate product functionalities, etc. The results of the survey are validated and enriched with indices derived by some more literature $[34-37,43,44]$. Again, the indices are measurable, as it happened for the characteristics. The result of this activiity is a set of eleven indices, collected in Table 3.

\subsubsection{Relating Prototyping Situations to Prototyping Activity Classes}

The relationships between prototyping situations and classes of prototyping activities are the base for the selection algorithm. We may establish this link because both elements deal with the same aspects. In fact, situation indices are nothing else than prototyping activity characteristics considered from the design point of view. The first two columns of Table 4 report this correspondence.

The other columns of Table 4 report average values of the indices related to the classes, computed thanks to these correspondences.

\subsubsection{The Selection Algorithm}

Now everything is ready for developing the selection algorithm. It is a very simple one at the moment, and this makes its implementation in a software package quite straightforward. The algorithm asks the designers to define the boundary of the problem to solve; they do this by

Table 2. Characteristics related to classes.

\begin{tabular}{|c|c|c|c|c|c|}
\hline Characteristic & $\mathbf{R P}$ & ARP & AVP & VP & MP \\
\hline Direct interaction & 1 & 0.1 & 0.95 & 0 & 0.6 \\
\hline Physical prototype & 1 & 0 & 1 & 0 & 1 \\
\hline Physical feedback & 1 & 0 & 0.9 & 0 & 0.7 \\
\hline Visual and audio feedback & 0 & 1 & 0.2 & 1 & 0.7 \\
\hline Real environment & 1 & 1 & 0 & 0 & 1 \\
\hline Functions for error recognition and recovery & 0.75 & 0.7 & 0.75 & 0.9 & 0.7 \\
\hline Functions implemented & 0.9 & 0.4 & 0.85 & 0.4 & 0.5 \\
\hline Time required for updates & 0.2 & 0.8 & 0.2 & 0.85 & 0.5 \\
\hline Cost required for updates & 0.2 & 0.8 & 0.2 & 0.9 & 0.7 \\
\hline Cost for tooling & 0.2 & 0.8 & 0.1 & 0.8 & 0.7 \\
\hline Cost for material & 0.2 & 1 & 0.2 & 1 & 0.7 \\
\hline Cost for prototyping & 0.3 & 0.9 & 0.4 & 0.95 & 0.9 \\
\hline Cost for set up & 0.45 & 0.95 & 0.3 & 1 & 0.7 \\
\hline Cost for working team & 0.2 & 0.2 & 0.2 & 0.2 & 0.2 \\
\hline Human operators & 0.2 & 0.8 & 0.5 & 1 & 0.8 \\
\hline Prototype building time & 0.1 & 0.75 & 0.3 & 0.75 & 0.5 \\
\hline Environment building time & 1 & 1 & 0.1 & 0.2 & 1 \\
\hline Dimensional and geometric accuracy & 0.8 & 0.2 & 0.9 & 0.9 & 0.2 \\
\hline
\end{tabular}


Table 3. Prototyping situation indices.

\begin{tabular}{ll}
\hline Index & Description \\
\hline $\begin{array}{l}\text { Direct interaction } \\
\text { Physical feedback }\end{array}$ & Need for direct user-prototype interaction \\
Total feedback & Amount of physical feedback to the user during the tests \\
Real environment & Potal amount of feedback \\
Error recognition and recovery & $\begin{array}{l}\text { Number of functions that recognize errors and are able to correct them autonomously } \\
\text { or with user's help }\end{array}$ \\
$\begin{array}{l}\text { Functions required } \\
\text { Prototype changing flexibility }\end{array}$ & $\begin{array}{l}\text { Need for modifying/changing prototype at low costs and time } \\
\text { Budget }\end{array}$ \\
Time & Total cost of prototyping activity \\
Human operators & Total time for prototyping activity \\
Tolerances & Available people for prototyping activity \\
\hline
\end{tabular}

Table 4. Relationships between situations and prototyping activities.

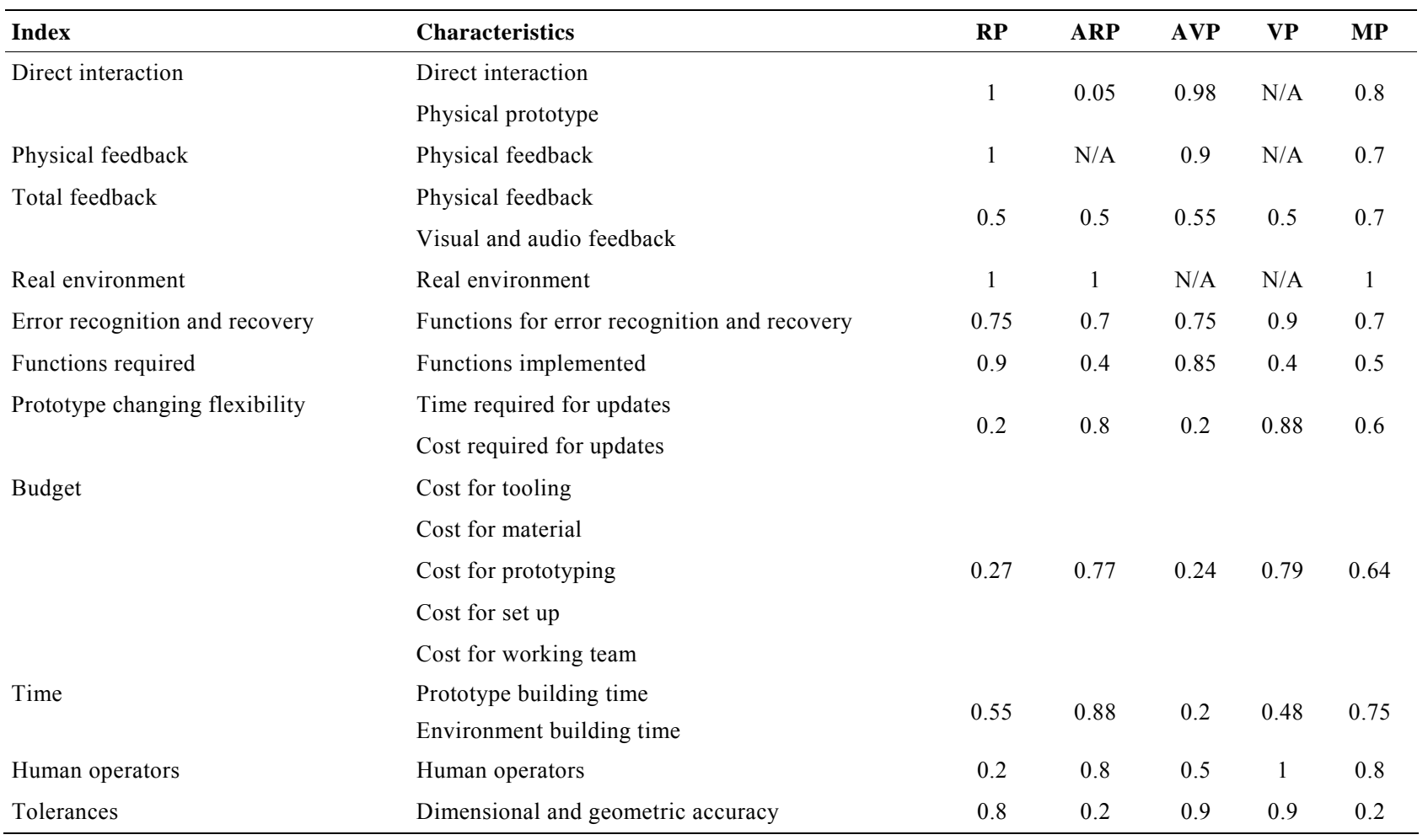

assigning weights representing the importance of the indices in their prototyping situations. The index values are collected in a vector. As before, values are in the range $[0,1]$. Then, the algorithm checks against blocking elements, the ones deriving from Boolean characteristics and indices. If an index weight is higher than 0.75 and the corresponding entry in Table 4 is equal to N/A, the whole column is set to N/A. For example, if the prototyping activities are required to have advanced physical feedback, the corresponding index weight is set to 0.9 . Given that ARP and VP classes have a N/A value in the corresponding entries of Table 4 , during the computation of the best prototyping activities for this particular case, all values of their columns are temporarily set to N/A.

Then, the index weights are multiplied by the entries of Table 4 and the sums of the results constitute the prototyping activity ranking for the specific situation.

\section{First Adoption in the Field}

Some experiences in the field have been the starting point for the validation of this research. Five prototyping situations have been selected to represent very different design scenarios, in order to verify if the selection of the 
right prototyping activities comes in the correct way. These scenarios are as follows.

1) Small furniture industry. The goal is to allow possible customers testing products against existing home furnishing. Then, real environment exploitation is a must. Moreover, time and budget are quite limited. At the same time, strict requirements exist regarding prototype flexibility, because product colors and details must be easily changed on the fly.

2) Big household appliance industry. The need is evaluating the remote control of a TV set. Time is limited, and direct interaction is firmly required. Being at the first design stage, flexibility and cheapness in prototype changing is another important aspect.

3) Mechanical workshop. The need is to test the mating of two components. Direct interaction and physical feedback are main concerns. Constraints about tolerances in prototype accuracy are present; moreover, almost all functions must be implemented in the prototype, because product purpose in this case is to act as a real joining element.

4) Cell phone industry. There is the need for testing a new model equipped with a touch screen. Major requirements are high flexibility in prototype changing, most of the functions implemented, feedback as complete as possible, limited costs.

5) Aviation industry. Testing of a new model of joystick. Real environment cannot be exploited, so it must be simulated during prototyping activities. There are not particular budget or time limitations, but direct interaction, implemented functions and total feedbacks are very important issues.

Table 5 reports the weights associated to the indices for the five prototyping situations. They allow running the software package implementing the algorithm.

Table 6 reports the results of the data elaboration.

Different prototyping activities are assigned to different prototyping situations, and all seems to happen in a correct way. Moreover, a ranking is generated for each situation, allowing the selection of alternative prototyping activities if the preferred ones should not be applicable for any reason.

\section{Discussion}

Some positive aspects of the research described up to now can be summarized as follows.

- The prototyping activity classification appears well defined, thanks to the characteristics allowing a clear description of them.

- The characteristics used for the classification and the indices associated to situations are general enough to make the resulting selection algorithm applicable in many different real scenarios.
Table 5. Index weights for the five prototyping scenarios.

\begin{tabular}{lccccc}
\hline \multirow{2}{*}{ Index } & \multicolumn{5}{c}{ Scenarios } \\
\cline { 2 - 6 } & $\boldsymbol{A}$ & $\boldsymbol{B}$ & $\boldsymbol{C}$ & $\boldsymbol{D}$ & $\boldsymbol{E}$ \\
\hline Direct interaction & 0.1 & 0.8 & 0.9 & 0.4 & 0.9 \\
Physical feedback & 0.1 & 0.8 & 0.95 & 0.2 & 0.9 \\
Total feedback & 0.8 & 0.85 & 0.95 & 0.8 & 0.8 \\
Real environment & 0.95 & 0.85 & 0.9 & 0.1 & 0.1 \\
Error recognition and recovery & 0.3 & 0.3 & 0.1 & 0.9 & 0.75 \\
Functions required & 0.6 & 0.85 & 0.95 & 0.95 & 0.9 \\
Prototype changing flexibility & 0.95 & 0.9 & 0.3 & 0.95 & 0.2 \\
Budget & 0.9 & 0.8 & 0.65 & 0.9 & 0.2 \\
Time & 0.95 & 0.85 & 0.8 & 0.95 & 0.3 \\
Human operators & 0.8 & 0.75 & 0.6 & 0.9 & 0.95 \\
Tolerances & 0.1 & 0.4 & 0.95 & 0.5 & 0.85 \\
\hline
\end{tabular}

Table 6. Results of the selection algorithm execution.

\begin{tabular}{cccccc}
\hline \multirow{2}{*}{ Rank } & \multicolumn{5}{c}{ Scenarios } \\
\cline { 2 - 6 } & $\boldsymbol{A}$ & $\boldsymbol{B}$ & $\boldsymbol{C}$ & $\boldsymbol{D}$ & $\boldsymbol{E}$ \\
\hline I & ARP (4.75) & MP (5.65) & RP (5.71) & VP (4.93) & AVP (4.84) \\
II & MP (4.69) & RP (5.20) & MP (5.36) & MP (4.90) & RP (4.80) \\
III & RP (3.51) & & & ARP (4.63) & MP (4.39) \\
IV & & & & RP (4.17) \\
V & & & & AVP (3.99) \\
\hline
\end{tabular}

- Current release of the selection algorithm is quite simple and its implementation in a software package is straightforward, replicable in different development environments.

Regarding some drawbacks, accounted for as future work, they are as follows.

- The prototyping activity classification has been derived from literature and with the help of three experts only. For this reason it cannot be intended as exhaustive. Many experiences in the field are still required to update/integrate the characteristic set and to refine the numerical values.

- At the moment, designers must describe their scenarios autonomously. This could be somehow dangerous, because they could not be aware of the implications of their claims. Some sort of expert system should be introduced to guide their involvement in the process.

- Looking at Table 6, some columns present values very close to each other. This happens because all starting values are in the range $[0,1]$. Maybe this needs to be revised.

\section{Conclusion}

The goal of this research has been to classify modern prototyping activities oriented to usability issues and to 
generate an automatic selection algorithm, given the peculiarities of application scenarios. Existing classifications have been considered at the beginning, and the highlight of their best and worst aspects allowed developing a set of eighteen characteristics, used to generate five classes of prototyping activities. Design situations where prototyping could be exploited have been described thanks to eleven indices. These indices, mapped with the eighteen characteristics, allow selecting the best prototyping activities case by case. Five experiences in the field started to prove the validity of the results. Some aspects of the research need to be focused on. The quantifications inside tables need to be improved by thinking about more objective and quantifiable methods, and refined by considering a wider set of experiences in the field; moreover, a more guided survey for designers should be developed, to help them in describing their scenario in an effective way. Finally, a more specific analysis is needed in order to really understand and take into the correct consideration the influence of prototyping timing in product development process. All these aspects need to be validated by real case studies performed in industries belonging to different domains. This will allow a better customization of the selected set of prototyping activities.

\section{Acknowledgements}

The authors would like to thank eng. Marco Amato for his help during the development of the research described in this paper.

\section{REFERENCES}

[1] M. Hertzum, "Images of Usability," International Journal of Human-Computer Interaction, Vol. 26, No. 6, 2010, pp. 567-600. doi:10.1080/10447311003781300

[2] A. Koca, M. Funk, E. Karapanos, A. Rozinat, J. Martens and A. Brombacher, "Soft Reliability: An Interdisciplinary Approach with a User-System Focus," Quality and Reliability Engineering International, Vol. 25, No.1, 2008, pp. 3-20. doi:10.1002/qre.937

[3] A. Umar and K. K. Tatari, "Appropriate Web Usability Evaluation Method during Product Development," Master Thesis, Software Engineering, School of Engineering, Blekinge Institute of Technology, Ronneby, 2008.

[4] Usability Set, 2011. http://www.usabilitynet.org

[5] J. Nielsen, "Usability Engineering," Academic Press, New York, 1993.

[6] A. Dix, J. Finlay, G. Abowd and R. Beale, "HumanComputer Interaction," 2nd Edition, Prentice Hall Europe, London, 1998.

[7] T. S. Andre, H. R. Hartson, S. M. Belz and F. A. McCreary, "The User Action Framework: A Reliable Foundation for Usability Engineering Support Tools," International Jour- nal of Human-Computer Studies, Vol. 54, No. 1, 2001, pp. 107-136. doi:10.1006/ijhc.2000.0441

[8] D. Lockton, D. Harrison and N. A. Stanton, "The Design with Intent Method: A Design Tool for Influencing User Behavior," Applied Ergonomics, Vol. 41, No. 3, 2010, pp. 382-392. doi:10.1016/j.apergo.2009.09.001

[9] H. Y. Kan, V. G. Duffy and C. Su, "An Internet Virtual Reality Collaborative Environment for Effective Product Design," Computer in Industry, Vol. 45, No. 2, 2001, pp. 197-213. doi:10.1016/S0166-3615(01)00093-8

[10] M. Fairen, P. Brunet and T. Techmann, "MiniVR: A Portable Virtual Reality Application," Computers \& Graphics, Vol. 28, No. 2, 2004, pp. 289-296. doi:10.1016/j.cag.2003.12.013

[11] S. H. Choi and A. M. M. Chan, "A Virtual Prototyping Application for Rapid Product Development," ComputerAided Design, Vol. 36, No. 5, 2004, pp. 401-412. doi:10.1016/S0010-4485(03)00110-6

[12] J. Verlinden, B. Smit and I. Hovath, "Case-Based Exploration of the Augmented Prototyping Dialogue to Support Design," Proceeding of the TMCE 2004, Lausanne, 12-16 April 2004, pp. 1-10.

[13] S. P. Lee, T. C. T. Qui, S. C. Loy and W. R. Pensyl, "Haptic Interaction in Augmented Reality," Proceeding of the 17th ACM International Conference on Multimedia, Vancouver, 2009, pp. 975-976.

[14] R. T. Azuma, “A Survey of Augmented Reality," Presence: Teleoperators and Virtual Environment, Vol. 6, No. 4, 1997, pp. 355-385.

[15] F. Bruno, F. Cosco, A. Angilica and M. Muzzupappa, "Mixed Prototyping for Product Usability Evaluation," Proceeding of the ASME 2010 International Design Engineering Technical Conferences \& Computers and Information in Engineering Conference, Montreal, 2010, pp. 1381-1390.

[16] P. Milgram and F. Kishino, "A Taxonomy of Mixed Reality Visual Displays," IEICE Transactions on Information System, Vol. E77-D, No. 12, 1994, pp. 1321-1329.

[17] T. Salloum, B. Anselmetti and K. Mawussi, "Design and Manufacturing of Parts for Functional Prototypes on FiveAxis Milling Machines," The International Journal of Advanced Manufacturing Technology, Vol. 45, No. 7-8, 2009, pp. 666-678. doi:10.1007/s00170-009-2013-Z

[18] F. P. W. Melchels, J. Feijen and D. W. Grijpma, “A Review on Stereolithography and Its Applications in Biomedical Engineering," Biomaterials, Vol. 31, No. 24, 2010, pp. 6121-6130. doi:10.1016/j.biomaterials.2010.04.050

[19] I. H. Song and S. C. Chung, "Synthesis of the Digital Mock-Up System for Heterogeneous CAD Assembly," Computers in Industry, Vol. 60, No. 5, 2009, pp. 285-295. doi:10.1016/j.compind.2008.09.004

[20] F. Bruno, A. Angilica, F. Cosco, M. L. Luchi and M. Muzzupappa, "Analysis of Video Tracking Techniques for Mixed Prototyping Applications," Proceeding of the IMProVe 2011, International Conference on Innovative Methods in Product Design, Venice, 15-17 June 2011, pp. 105-113. 
[21] M. Bordegoni, U. Cugini, G. Caruso and S. Polistena, "Mixed Prototyping for Product Assessment: A Reference Framework," International Journal on Interactive Design and Manufacturing, Vol. 3, No. 3, 2009, pp. 177187. doi:10.1007/s12008-009-0073-9

[22] D. Frohlich, "The History and the Future of Direct Manipulation," Information System Laboratories, HP Laboratories, Bristol, 1993.

[23] C. K. Chua, K. F. Leong and C. S. Lim, "Rapid Prototyping: Principles and Applications," 3rd Edition, World Scientific Publishing, New York, 2010.

[24] D. T. Pham and R. S. Gault, "A Comparison of Rapid Prototyping Technologies," International Journal of Machine Tools \& Manufacture, Vol. 38, No. 10-11, 1998, pp. 1257-1287. doi:10.1016/S0890-6955(97)00137-5

[25] S. Filippi, D. Barattin, M. Amato and R. Tozzi, "An Augmented Reality Based Application for Furnishing Configuration and Evaluation," Proceeding of the ASME 2011 World Conference on Innovation Virtual Reality, Milan, 27-29 June 2011, pp. 19-29. doi:10.1115/WINVR2011-5530

[26] Aurasma, 2012. http://www.aurasma.com

[27] G. Papagiannakis, S. Schertenleib and B. O'Kennedy, "Mixing Virtual and Real Scenes in the Sites of Ancient Pompeii," Computer Animation and Virtual Worlds, Wiley Interscience, Vol. 16, No. 1, 2005, pp. 11-24. doi: $10.1002 /$ cav. 53

[28] A. Nagar and H. Tawfik, "A Multi-Criteria Based Approach to Prototyping Urban Road Networks," Informing Science and Information Technology, Vol. 4, 2007, pp. 749-756.

[29] R. Yang and L. Yan, "The Design of a 3D Flight Simulation and Monitoring Platform," Advanced Materials Research, Vol. 466-467, 2012, pp. 971-975.

[30] Autodesk, 2011. http://www.autodesk.it/adsk/servlet/home

[31] Blender, 2011. http://www.blender.it

[32] Tutorials for Blender 3D, 2011. http://www.tutorialsforblender3d.com/Game_Engine/Tut orials index.html

[33] Sébastien "VR Geek” Kuntz, 2011. http://cb.nowan.net/blog/2008/04/

[34] A. Armillotta, "Selection of Layered Manufacturing Techniques by an Adaptive AHP Decision Model," Robotics and Computer-Integrated Manufacturing, Vol. 24, No. 3,
2008, pp. 450-461. doi:10.1016/j.rcim.2007.06.001

[35] S. Khrais, T. Al-Hawari and O. Al-Araidah, "A Fuzzy Logic Application for Selecting Layered Manufacturing Techniques," Expert System with Applications, Vol. 38, No. 8, 2011, pp. 10286-10291. doi:10.1016/j.eswa.2011.02.061

[36] S. H. Masood and A. Soo, "A Rule Based Expert System for Rapid Prototyping System Selection," Robotics and Computer-Integrated Manufacturing, Vol. 18, No. 3-4, 2002, pp. 267-274. doi:10.1016/S0736-5845(02)00017-0

[37] M. Yurdakul, "AHP as a Strategic Decision-Making Tool to Justify Machine Tool Selection," Journal of Materials Processing Technology, Vol. 146, No. 3, 2004, pp. $365-$ 376. doi:10.1016/j.jmatprotec.2003.11.026

[38] S. Kim and A. K. Dey, "AR Interfacing with Prototype 3D Applications Based on User-Centered Interactivity," Computer-Aided Design, Vol. 42, No. 5, 2010, pp. 373 386. doi:10.1016/j.cad.2008.10.009

[39] C. Kim, C. Lee, M. R. Lehto and M. H. Yun, "Evaluation of Customers Impressions Using Virtual Prototypes in the Internet Environment," International Journal of Industrial Ergonomics, Vol. 41, No. 2, 2011, pp. 118-127. doi:10.1016/j.ergon.2010.12.006

[40] M. Fumarola and R. Poelman, "Generating Virtual Environments of Real World Facilities: Discussing Four Different Approaches," Automation in Construction, Vol. 20, No. 3, 2011, pp. 263-269. doi:10.1016/j.autcon.2010.08.004

[41] J. Ye, R. I. Campbell, T. Page and K. S. Badni, “An Investigation into the Implementation of Virtual Reality Technologies in Support of Conceptual Design," Design Studies, Vol. 27, No. 1, 2006, pp. 77-97. doi:10.1016/j.destud.2005.06.002

[42] J. Verlinden, A. de Smit, A. W. J. Peeters and M. H. van Gelderen, "Development of a Flexible Augmented Prototyping System," Journal of WSCG, Vol. 11, No. 3, 2003, pp. 496-503.

[43] R. S. Kalawsky, S. T. Bee and S. P. Nee, "Human Factors Evaluation Techniques to Aid Understanding of Virtual Interfaces," BT Technology Journal, Vol. 17, No. 1, 1998, pp. 128-141. doi:10.1023/A:1009687227736

[44] M. Braglia and A. Petroni, "A Management-Support Technique for the Selection of Rapid Prototyping Technologies," Journal of Industrial Technology, Vol. 15, No. 4, 1999, pp. 1-6. 